\title{
ESTUDO DA TERMOLUMINESCEENCIA NATURAL DO QUARTZITO, EM UMA ZONA MINERALIZADA EM OURO E URANNIO (CANAVIEIRAS, JACOBINA, BAHIA)
}

\author{
JORGE FERREIRA* e DANIEL NORDEMANN**
}

\begin{abstract}
The quartzite of the Canavieiras gold mine (Jacobina, Bahia, Brazil) exhibits a high temperature peak $(280 \pm 2)^{\circ} \mathrm{C}$ in its natural thermoluminescence glow curve. It was experimentally determined that the amount of light in this peak could be reproduced to within $5 \%$ for grain sizes between 80 and 100 mesh $(0.149$ and $0.177 \mathrm{~mm})$.

A positive correlation was found between the amount of this thermoluminescence and the uranium content with saturation beginning to be detected at the maximum (700 ppm) amounts of uranium found.

Assuming that the thermoluminescence peak used is at a temperature high enough to permit accumulation and stability at room temperatures, the thermoluminescence found could have accumulated in (12.1 \pm 0.3$) \times 10^{6}$ years. This may date a complete liberation of trapped electrons in conjunction with a well documented initiation of weathering.
\end{abstract}

INTRODUÇÃo A mina de ouro em Canavieiras está situada na Serra da Jacobina, $6 \mathrm{~km}$ ao sul da cidade do mesmo nome (Estado da Bahia). Essa serra faz parte da cadeia marginal da Cordilheira do Espinhaço, composta de rochas do embasamento cristalino Pré-Cambriano.

A ocorrência de urânio em Canavieiras foi descoberta por volta de 1953 (Bateman, 1958), sob forma de uraninita $\left(\mathrm{U}_{3} \mathrm{O}_{8}\right)$. Mais tarde, porém, verificou-se a existência de uma correlação entre ouro, pirita e minerais radioativos, mostrando-se inclusive a existência de uma maior concentração de urânio no nível piritoso e em torno de $0,006 \%$ (White, 1957).

Acredita-se que a mineralização em Canavieiras seja de origem hidrotermal (White, 1964). Assim sendo, camadas de quartzito e conglomerados associados a ouro, urânio e pirita têm sido encontradas em vários lugares na Serra de Jacobina.

TERMOLUMINESCENGIA A termoluminescência $(T L)$ é a emissão em forma de luz da energia armazenada por uma determinada substância quando aquecida. Esse fenômeno tem sido observado em sólidos não-condutores e tem sido sugerido como base de técnica de datação de rochas e minerais. Devido à presença de elementos radioativos naturais, os quais funcionam como fonte interna de radiação ionizante, supõe-se que os elétrons excitados por essa radiação sejam aprisionados em estados metastáveís. Na ocasião em que uma energia adicional (aquecimento) for cedida ao cristal, os èlétrons se tornarão livres, podendo ser recapturados por armadilhas ou se recombinarem com uma lacuna, emitindo luz. Fenômeno idêntico é observado nos quartzitos da mina de Canavieiras, os quais possuem radioatividade natural, tendo acumulado elétrons com o tempo.

A emissão de luz foi registrada em aparelho leitor de $T L$ convencional, sendo a função temperatura-tempo previamente estabelecida por uma fonte programada (Kepco Power Supply), fabricada pela Jarrel Ash. Como elemento aquecedor, foi utilizada uma

* Pesquisador do Programa de Pesquisa e Pós-Graduação em Geofísica do Instituto de Geociências, Universidade Federal da Bahia

**Perito da UNESCO (Projeto BRA-034/562 do POND-UNESCO), Professor Visitante da UFBa 
lâmina de níquel da baixa capacidade térmica, e todas as medidas foram realizadas durante um minuto e vinte e três segundos, a uma razão constante de aquecimento de $4,3^{\circ} \mathrm{C} / \mathrm{s}$, a partir da temperatura ambiente.

Parte experimental As amostragens foram precedidas por estudos dos níveis da radiação gama total no interior da mina (gamametria). Foi utilizado um cintilômetro por. tátil tipo SPP3, com o detetor envolvido em folha de chumbo de $2 \mathrm{~mm}$ de espessura, e colocado diretamente sobre a rocha a ser medida (Ferreira, 1973), permitindo assim minimizar os efeitos das contribuições dos emissores gama externos ao ponto da rocha medido. Dessa forma, foram levantados perfis de atividade gama total ao longo das damas* 1 e 2, tomando-se por base os níveis "Piritoso" e "Liberino", nos quais a amostragem foi efetuada.

Foi realizado um estudo sistemático da variação na intensidade de luz emitida pela amostra de quartzito, após diferentes tempos de pulverização (90 a $180 \mathrm{~s}$ ).

Os resultados experimentais (Fig. 1) mostram que as maiores variações na altura do pico $T L$ para iguais massas de amostra $(10 \mathrm{mg})$, ocorrem com os grãos triturados mais finos que $0,125 \mathrm{~mm}(115 \mathrm{mes} h)$. Visando minimizar os efeitos da não-transparência do-



Figura 1 - Variação da resposta termoluminescente em função do diametro dos grãos, para iguais pesos de amostras (J15)

*Colunas de sustentação das galerias construídas durante as escavações da rocha 
cristal à luz, assim como reflexões internas dos grãos da amostra, e evitar maiores influências de trituração, foram utilizados, neste trabalho, os cristais com dimensões entre $0,177 \mathrm{e}$ $0,149 \mathrm{~mm}(80$ a $100 \mathrm{mesh})$. Essa escolha se deve ao fato de que, nessa faixa, a altura do pico na curva de emissão não é sensivelmente afetada pelo tamanho do grão, nem pelo tempo de trituração. Graças a esse estudo, todas as curvas de emissão, representativas de diferentes pontos de uma mesma amostra, apresentam boa reprodutibilidade e dispersão inferior a $5 \%$ (desvio-padrão das medidas), garantindo a validade das medidas de $T L$ (Fig. 2).

Os teores dos principais elementos radioativos presentes nas amostras em estudo foram determinados por espectrometria gama em laboratório. $\mathrm{Na}$ obtenção dos espectros, utilizaram-se os seguintes equipamentos:

cadeia de contagem-gama de baixo ruido de fundo, composta de detetor $\mathrm{NaI}$ (Tl) $(10 \times 8 \mathrm{~cm})$

analisador de pulsos de 1024 canais (tipo 540 1B Hewlett Packard);

teleimpressora para registro numérico dos espectros;

fotomultiplicadora com janela de quartzo;

castelo de chumbo de $16 \mathrm{~cm}$ de espessura.

Todo o sistema foi calibrado para se obter uma repartição de $10 \mathrm{keV}$ por canal, e os espectros obtidos permitiram determinar, sem tratamento químico, as concentrações

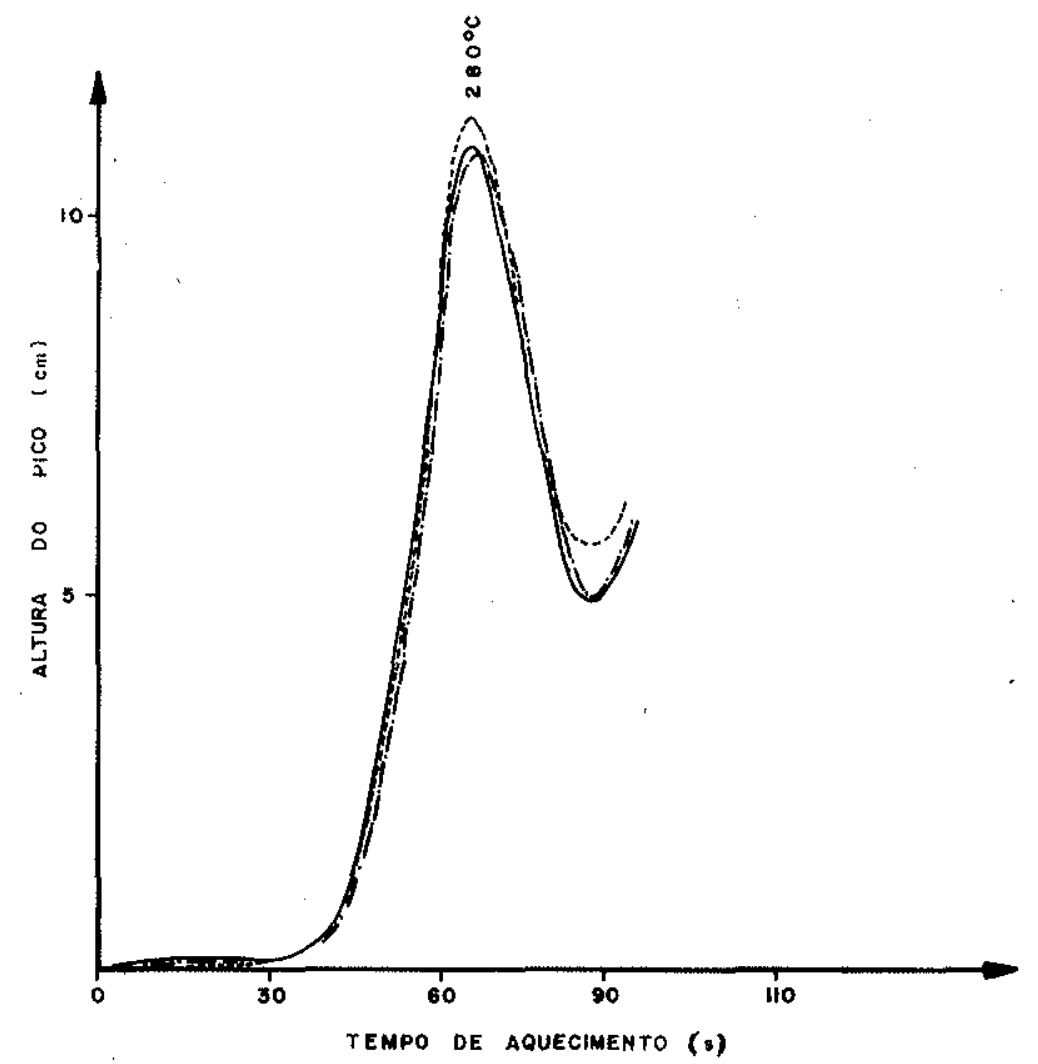

Figura 2 - Reprodutibilidade das curvas de emissão natural para iguais pesos de amostras de quartzito. A dimensão dos cristais usados na obtenção desta figura está compreendida entre $0,177 \mathrm{~mm}$ (80 mesh) e $0,149 \mathrm{~mm}(100$ mesh). Amostra $\mathrm{J} 7$, perfil 1, dama 1 
dos elementos radioativos urânio, tório e potássio. $\mathrm{O}$ método das tangentes foi utilizado nessas avaliações, além dos rendimentos de deteção determinados por Nordemann (1966).

IRRADIAÇÃO IN SITU DO MATERIAL ESTUDADO Visando estimar o percurso alcançado pelas principais radiações no quartzito e a energia liberada por uma massa (m) de amostra, foi realizada uma análise coerente das energias associadas às emissões, levando-se em conta as abundâncias e os esquemas de decaimento dos radionuclídeos. Admitiu-se cada família radioativa em equilíbrio secular. $O$ cálculo da energia liberada foi determinado utilizando-se a expressão:

$$
E_{T}(\mathrm{U}, \mathrm{Th}, \mathrm{K})=\left(Q_{i} \cdot C_{i}\right) \cdot m\left(\mathrm{erg} \cdot \text { ano }^{-1}\right),
$$

onde $Q_{i}$ é a energia específica liberada, $\mathrm{em} \mathrm{erg} \cdot \mathrm{g}^{-1} \cdot \mathrm{ano}^{-1}$, de urânio, tório e potássio; $C_{i}$ representa o teor do elemento (i) na amostra, em $\mathrm{g} \cdot \mathrm{g}^{-1}$; e $m$ é a massa de amostra usada nas medidas de $T L(10 \mathrm{mg})$.

$\mathrm{O}$ percurso das partículas alfa foi estimado em relação ao ar e, posteriormente, calculado para o quartzito, através da expressão

$$
P=\left(\rho / \rho_{Q}\right) C,
$$

onde $\rho$ representa a densidade do ar, $\rho_{Q}$ a densidade do quartzito e $C$ o percurso das partículas alfa no ar.

De maneira idêntica se procedeu para calcular o percurso das partículas beta em relação ao alumínio. $\mathrm{O}$ alcance máximo atingido pelas partículas alfa no quartzito é de $3,7 \cdot 10^{-3} \mathrm{~cm}(37 \mu \mathrm{m})$, correspondendo a uma energia de 7,680 MeV, e tendo como emissor principal o ${ }_{84} \mathrm{Po}^{214}(\mathrm{RaC})$. Para a radiação beta, o alcance máximo foi calculado como sendo igual a $2,8 \cdot 10^{-1} \mathrm{~cm}\left(2,8 \cdot 10^{3} \mu \mathrm{m}\right)$, correspondendo a uma energia máxima de $3,17 \mathrm{MeV}$, e tendo como emissor o $\mathrm{Bi}^{214}$. A interação dos raios gama com a matéria é diferente, em virtude da diferença no poder de penetração, e das leis que regem sua absorção. Para o quartzito, o inverso do coeficiente de absorção corresponde a um comprimento de $10,4 \mathrm{~cm}$, para a maior energia gama emitida pelo $\mathrm{Tl}^{208}$ ( $\mathrm{Th} \mathrm{C}^{\prime \prime}$ ). Devido ao caráter exponencial dessa radiação não se pode definir um percurso correspondente a $100 \%$ da energia da radiação incidente sobre a matéria, mas sabe-se que $99 \%$ desta processa-se a uma distância, contada a partir da fonte emissora, de 4,6 vezes o inverso do coeficiente de absorção.

Nas Tabs. I e II pode-se observar que, para um meio homogêneo, a radiação alfa emitida pelo urânio é o principal contribuinte para a $T L$; muito embora a radiação alfa do tório tenha um poder de penetração maior, sua energia dissipada sob forma de radiação ionizante é nitidamente inferior.

Assim, a $T L$ de um pequeno volume de amostra nesse meio depende principalmente dos emissores locais da amostra em si, e não da radiação gama que vem de mais longe. De uma maneira geral, pode-se dizer que a totalidade das energias liberadas pela família do $U^{238}$, através das radiaçð̃es alfa e beta, são dissipadas em esferas concêntricas tendo comó raios os alcances máximos de $3,7 \cdot 10^{-3}$ e $2,8 \cdot 10^{-1} \mathrm{~cm}$, respectivamente. $\mathrm{E}$, também, os $99 \%$ da energia da radiação gama para a mesma família, são dissipados em uma esfera concêntrica com as duas primeiras, e de raio igual a $48 \mathrm{~cm}$.

Em geral, para as medidas de $T L$ natural e espectrometria gama, foram recolhidas, em cada ponto dos perfis, amostras de forma cúbica, com $7,3 \mathrm{~cm}$ de lado, a uma distancia superior ao percurso da radiação beta de maior energia $\left(3,17 \mathrm{MeV}, \mathrm{Bi}^{214}\right)$. Com esse resultado, pode-se dizer que a $T L$ de um pequeno volume de amostra, em meio homogêneo, resulta das irradiações alfa e beta de curto alcance. 
Tabela I - Energia total por elemento e percurso atingido pelas principais emissð̃es radioativas no quartzito

\begin{tabular}{|c|c|c|c|c|c|}
\hline $\begin{array}{l}\text { Elementos } \\
\text { radioativos }\end{array}$ & Radiaçóes & $\begin{array}{c}\text { Energia média } \\
\text { dissipada por } \\
\text { decaimento }(\mathrm{MeV})\end{array}$ & $\begin{array}{c}\text { Fração da } \\
\text { energia } \\
\text { total }(\%)\end{array}$ & $\begin{array}{l}\text { Percurso da radiação } \\
\text { de maior energia }(\mathrm{cm})\end{array}$ & $\begin{array}{l}\text { Percurso } \\
\text { em }(\mu \mathrm{m})\end{array}$ \\
\hline \multirow{3}{*}{$\mathrm{U}^{238}$} & Alfa & 42,8 & 91,1 & $3,7 \cdot 10^{-3}$ & 37 \\
\hline & Beta & 2,4 & 5,1 & $2,8 \cdot 10^{-1}$ & $28 \cdot 10^{2}$ \\
\hline & Gama & 1,8 & 3,8 & 9,2 & $92 \cdot 10^{3}$ \\
\hline Total & - & 47,0 & 100,0 & - & - \\
\hline \multirow{3}{*}{$\mathrm{Th}^{232}$} & Alfa & 35,7 & 92,2 & $4,3 \cdot 10^{-3}$ & 43 \\
\hline & Beta & 1,6 & 4,2 & $2,4 \cdot 10^{-1}$ & $24 \cdot 10^{2}$ \\
\hline & Gama & 1,4 & 3,6 & 10,4 & $104 \cdot 10^{3}$ \\
\hline Total & - & 38,7 & 100,0 & - & - \\
\hline \multirow{2}{*}{$K^{40}$} & Beta & 0,4 & 66,7 & $0,8 \cdot 10^{-1}$ & $8 \cdot 10^{2}$ \\
\hline & Gama & 0,2 & 33,3 & 7,5 & $75 \cdot 10^{3}$ \\
\hline Total & & 0,6 & 100,0 & - & - \\
\hline
\end{tabular}

Observação: no cálculo feito para a radiação gama foi considerado o comprimento correspondente a uma absorção de $99 \%$ da radiação $(x=4,6 \lambda)$

Tabela II - Contribuição dos principais elementes radioativos à termoluminescência para $10 \mathrm{mg}$. de amostras

\begin{tabular}{|c|c|c|c|c|c|}
\hline \multirow[b]{2}{*}{$\begin{array}{l}\text { Elemento } \\
\text { radioativo }\end{array}$} & \multicolumn{3}{|c|}{ Radiaçz̃es } & \multirow[b]{2}{*}{$\begin{array}{l}\text { Total por } \\
\text { elemento } \\
(\mathrm{MeV} / \mathrm{s})\end{array}$} & \multirow[b]{2}{*}{$\begin{array}{c}\text { Fração das contribuições } \\
\text { por elemento }(\%)\end{array}$} \\
\hline & $\begin{array}{c}\text { Alfa } \\
(\mathrm{MeV} / \mathrm{s}) \\
\end{array}$ & $\begin{array}{c}\text { Beta } \\
(\mathrm{MeV} / \mathrm{s})\end{array}$ & $\begin{array}{c}\text { Gama } \\
(\mathrm{MeV} / \mathrm{s})\end{array}$ & & \\
\hline$U^{238}$ & 9,0 & 1,5 & 0,4 & 10,9 & 78,9 \\
\hline $\mathrm{Th}^{232}$ & 2,2 & 0,3 & 0,1 & 2,6 & 18,9 \\
\hline$\overline{\mathrm{K}^{4}} \overline{0}$ & - & 0,2 & 0,1 & 0,3 & 2,2 \\
\hline $\begin{array}{l}\text { Total por } \\
\text { radiaçăo }\end{array}$ & 11,2 & 2,0 & 0,6 & 13,8 & 100,0 \\
\hline $\begin{array}{l}\text { Fração por } \\
\text { radiação }(\%)\end{array}$ & 81,2 & 14,4 & 4,4 & 100,0 & - \\
\hline
\end{tabular}

Os cálculos foram realizados com referência à amostra $\mathrm{J9}$ perfil 1 , dama 1 . As concentraçð̃es de urânio, tório e potássio para esta amostra foram calculadas por espectometria gama, com os seguintes resultados: U, $17 \mathrm{ppm}$; .Th, $15 \mathrm{ppm}$; e K, 0,81\%.

Observaçôes:

$\mathrm{l}$ g de $\mathrm{U}$ é equivalente a $1,23 \cdot 10^{4} \mathrm{dps}$ de urânio 238
$\mathrm{lg}$ de Th é equivalente a $4,1 \cdot 10^{3} \mathrm{dps}$ de tório 232
$\mathrm{l} \mathrm{g}$ de $\mathrm{K}$ é equivalente a $31,45 \mathrm{dps}$, de potássio 40

CALIBRAÇÃO ÓPTICA DO APARELHO LEITOR DE TL U ma lâmpada incandescente, convenientemente alimentada, foi utilizada como fonte calibrada de luz, dada à falta absoluta de uma fonte-padrão. Sabendo-se ser $4 \pi$ a potência emitida por essa lâmpada sob forma de luz, a potência de alimentação $0,528 \mathrm{~W}$, o rendimento luminoso 7,9 lúmen/W e a equivalência energética do lámen, para um comprimento de onda de $0,556 \mathrm{~nm} ;(1 \mathrm{~W}=621$ lúmen $)$, foi possível obter, no registrador que compõe o sistema 
leitor da $T L$, desvios, em centímetros, comparáveis com aos obtidos quando das medidas da $T L$ natural. Além disso, foi considerada também a relação entre os ângulos sólidos, subtendidos pelo fotocátodo a partir da posição onde se encontra a lâmpada $(2,0 \mathrm{~m})$, e a partir da lâmina de níquel onde a amostra é colocada para aquecimento posterior ( $3,0 \mathrm{~cm}$ do fotocátodo). Como resultado, a sensibilidade do aparelho de deteção da $T L$ foi calculada em $11,1 \mathrm{~cm} / \mu \mathrm{W}$, ou seja, $9 \cdot 10^{-2} \mu \mathrm{W} / \mathrm{cm}$ de desvio do registrador. As curvas de emissão da $T L$ natural foram descritas durante $50,4 \mathrm{~s}$, podendo-se expressar suas áreas em unidades de energia. Para os cálculos, considerou-se a forma do pico semelhante à de um triângulo, resultando:

$$
A=22,7 \text { ergs. }
$$

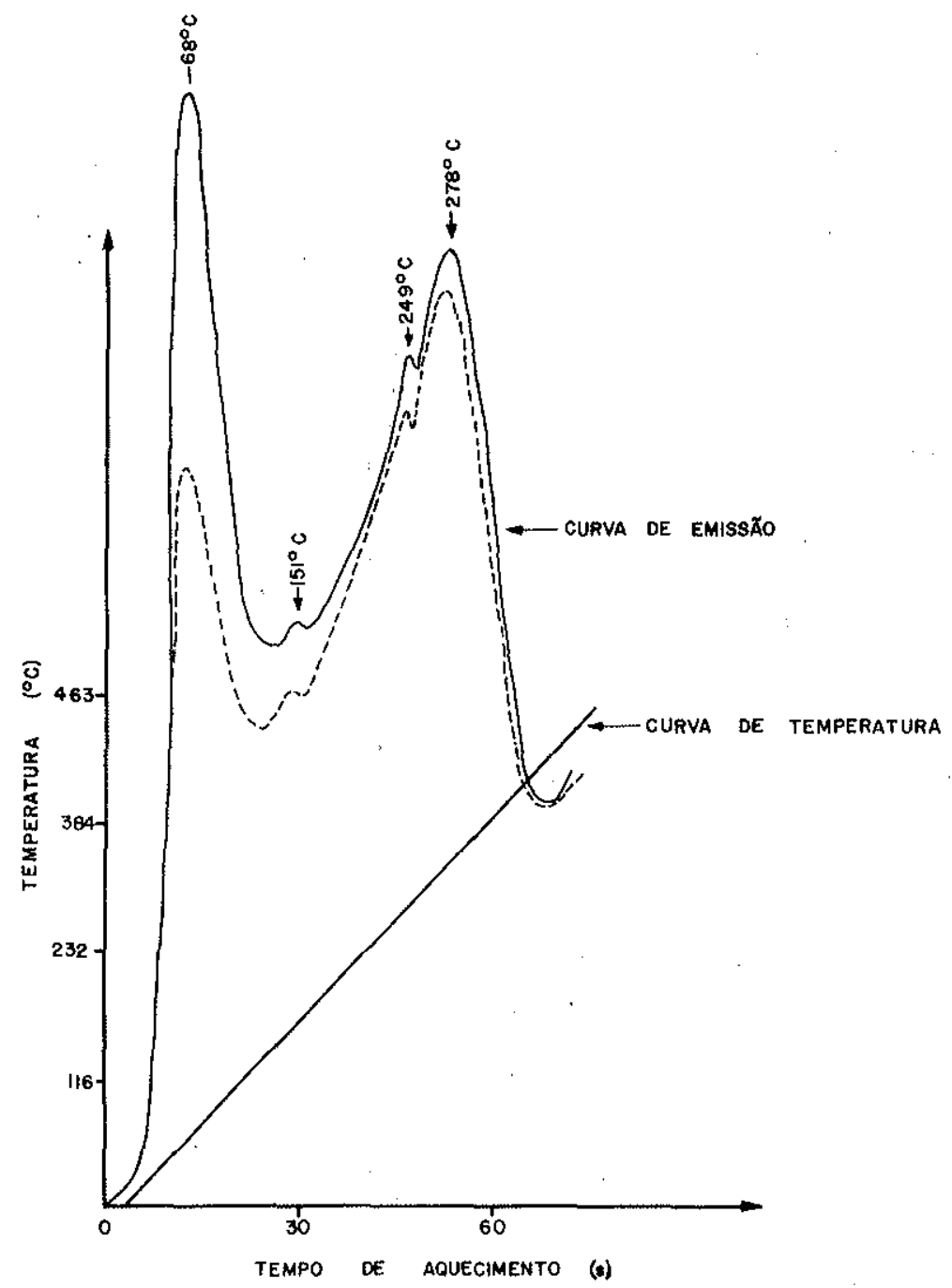

Figura 3 - Reprodutibilidade das curvas de emissão do quartzito, irradiado durante 30 min por fonte de raios $X$. As temperaturas dos máximos de emissão foram de $68 \pm 2,151 \pm 2,249 \pm 1 \mathrm{e}$ $278 \pm 2$ para uma mesma amostra (J9). Cada leitura foi feita $10 \mathrm{~min}$ após a irradiação 
TERMOLUMINESGENAIA INDUZIDA POR MEIO DE RAIOS X; ESTIMATIVA DO RENDIMENTO DE AGUMULAÇÃO DA TL NO QUARTZITO Entende-se por "rendimento de acumulação" a relação da energia luminosa emitida por uma massa $(m)$ de uma substância termoluminescente com a energia proveniente da radiação à qual foi exposta. Para avaliá-lo, foi necessário inicialmente submeter as amostras a um pré-tratamento térmico durante $20 \mathrm{~min}$ a $300^{\circ} \mathrm{C}$, a fim de drenar a $T L$ natural existente. Um aparelho gerador de raios X (Rigaku Denki Co, Ltd.) com ânodo de cobre foi utilizado durante as irradiações, aplicando-se uma tensão de $35 \mathrm{kV}$, superior à tensão crítica de aceleração dos elétrons, para a excitação da radiação característica da série $K\left(V_{K}=9 \mathrm{kV}\right)$, e uma corrente de $20 \mathrm{~mA}$. Cada amostra $(10 \mathrm{mg})$ foi colocada em recipiente plástico e, em seguida, irradiada a uma distância fixa de $12,0 \mathrm{~cm}$ da fonte. As medidas de $T L$ artificiais foram feitas dez minutos após as irradiações, e as curvas de emissão exibiram quatro picos, cujas temperaturas de seus máximos foram, respectivamente, $68 \pm 2,151 \pm 2$, $249 \pm 1$ e $278 \pm 2{ }^{\circ} \mathrm{C}$ (Fig. 3).

Com base nos resultados experimentais, foi utilizada a dose equivalente de $1,95 \cdot 10^{7}$ erg, necessária para reproduzir um pico de $1 \mathrm{~cm}$ de altura correspondendo à emissão de $22,7 \mathrm{erg}$. Em conseqüência, o rendimento de acumulação foi avaliado em $1,2 \cdot 10^{-5}$. Para comparar a $T L$ natural com a $T L$ artificial entre picos de mesma altura, estima-se a energia dissipada na amostra por unidade de tempo. Assim, devido às condições utilizadas nas irradiações, e à produção de raios (rendimento menor que $1 \%$, Cullity, 1967), a potência do feixe incidente no anticátodo foi calculada em $7 \mathrm{~W}$ aproximadamente. Por outro lado, levando-se em conta sua absorção no ar e no material, de espessura $L$, a fração do feixe absorvida pela amostra foi avaliada como segue:

onde

$$
P=\frac{S}{2 \pi R^{2}} K P_{0} \cdot e^{-\mu_{a r} R}\left[1-e^{-\mu\left(S \mathrm{~S}_{2}\right) L}\right]
$$

$$
\frac{S}{2 \pi R^{2}}
$$

representa o ângulo sólido subtendido pela amostra a partir do feixe de raios $\mathrm{X}$ emitido no anticátodo;

$K$, fração do feixe de raios $\mathrm{X}$, transmitida através da janela de berilo do tubo gerador;

$P_{0}$, potência do feixe emitido no anticátodo;

$\mu_{a r}$, coeficiente médio de absorção do feixe de raios $\mathrm{X}$ no ar;

$e^{-\mu_{a r R}}$, fração residual do feixe, após percorrer a distância $R$ no ar.

Os coeficientes de absorção do feixe de raios $\mathrm{X}$ no ar e na sílica foram considerados iguais a 9,8 e $80 \mathrm{~cm}^{2} / \mathrm{g}$, respectivamente, para o comprimento de onda da radiação característica do cobre, $\operatorname{Cu} K_{\alpha}(\lambda=1,542 \AA)$. Em consequêencia, a energia dissipadá na amostra foi estimada em $2,17 \cdot 10^{4} \mathrm{erg} \cdot \mathrm{s}^{-1}$, o que possibilitou a obtenção da curva de calibração de $T L$ vérsus dose absorvida (Fig. 4).

A escala superior da abscissa, na Fig. 4, representa o tempo de exposição a que foram submetidas as amostras aos raios $\mathrm{X}$, enquanto que a escala inferior representa a dose absorvida (ergs). Na escala vertical estão representados os valores médios da altura do pico de mais alta temperatura detetado após a irradiação $278 \pm 2{ }^{\circ} \mathrm{G}$.

Discussåo e interpretaçăo dos resultados As amostras de quartzito exibiram, em sua curva de emissão, um pico de $T L$ natural, cuja temperatura foi avaliada em $280 \pm 2{ }^{\circ} \mathrm{C}$. Os resultados do estudo da $T L$ natural e as medidas de espectrometria gama são vistos nas Tabs. III e IV. 


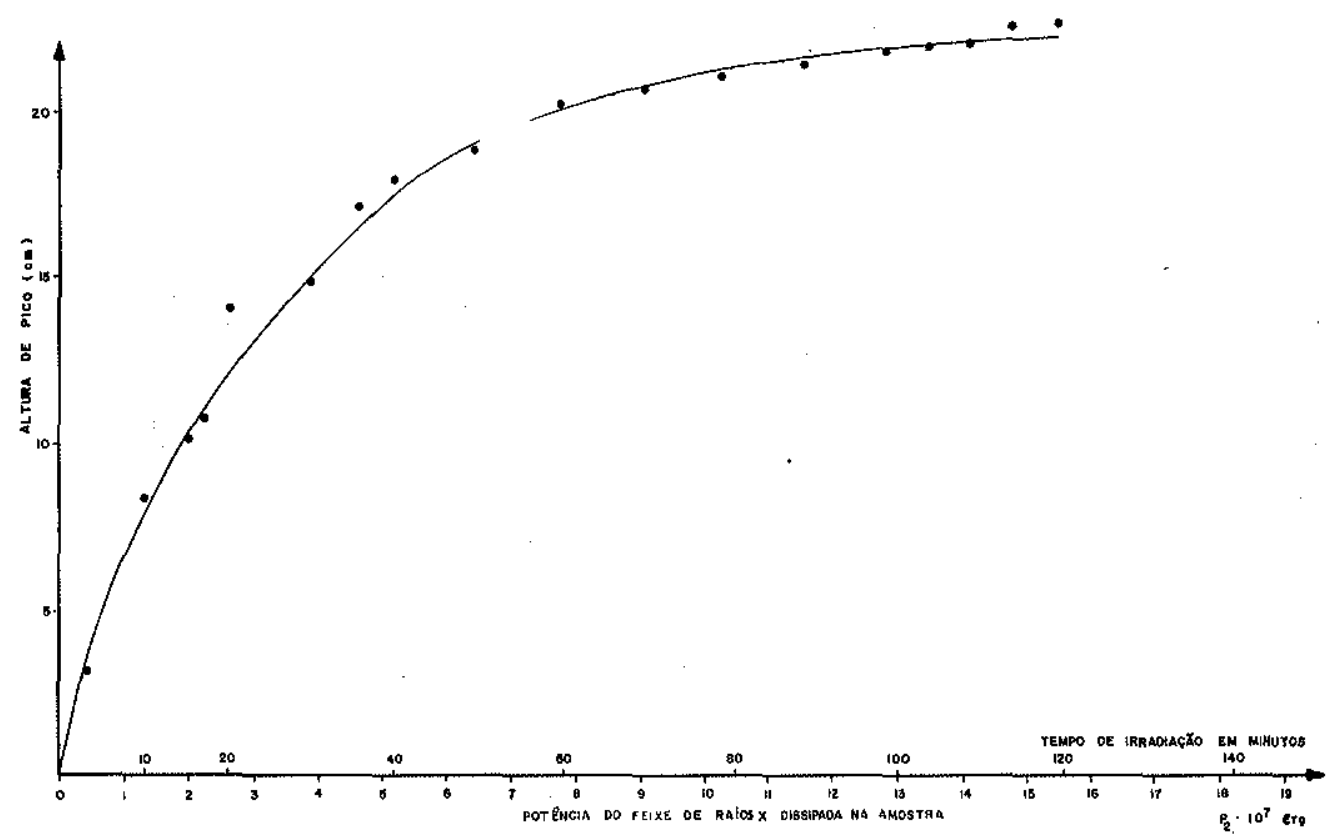

Figura 4 - - Gurva de calibração da $T L$ induzida pelos raios $\mathrm{X}$, em função da potência do feixe dissipado na amostra (J9), por tempo de exposição diferente. Para a excitação da radiação característica da série $\mathrm{K}$ no tubo de raios $\mathrm{X}$, foi aplicada uma tensão superior ao valor crítico $\left(V_{\mathrm{K}}\right)$ de $35 \mathrm{kV}$ e uma corrente eletrônica de $20 \mathrm{~mA}$

Tabela III - Medidas de termoluminescência natural e avaliação de dose para as amostras do perfil 1, dama 1, levandomse em consideração as famílias do $\mathrm{U}^{238}, \mathrm{Th}^{232}$ e $\mathrm{K}^{40}$. Temperatura do pico de $T L$ natural $=280 \pm 2{ }^{\circ} \mathrm{C}$

\begin{tabular}{|c|c|c|c|c|c|c|c|}
\hline Amostras & $\begin{array}{l}\text { Atividade } \\
\text { (SPP3) } \\
\text { in laco }\end{array}$ & $\begin{array}{c}\text { Termolum } \\
\text { H (cm) }\end{array}$ & $\begin{array}{l}\text { ếncia natural } \\
\text { Área }\left(\mathrm{mm}^{2}\right)\end{array}$ & $\begin{array}{l}\text { Urânjo } \\
\text { (ppm) }\end{array}$ & $\begin{array}{l}\text { Tório } \\
\text { (ppm) }\end{array}$ & $\begin{array}{l}\text { Potássio } \\
(\%)\end{array}$ & $\begin{array}{l}\text { Dose de radiação } \\
\text { natural }{ }^{* *} \text { (erg/ano) }\end{array}$ \\
\hline J1 & 120 & 3,8 & 460 & $3,0 \pm 0,5$ & $4,0 \pm 0,4$ & $0,29 \pm 0,03$ & 1,28 \\
\hline J3 & 200 & 4,9 & 563 & $3,0 \pm 0,6$ & $14,0 \pm 1,5$ & $0,3.2 \pm 0,03$ & 2,12 \\
\hline 54 & 270 & 6,1 & 700 & ـ- & - & - & - \\
\hline J5 & 350 & 6,6 & 680 & $s, 0 \pm 1,0$ & $15,0 \pm 1,5$ & $0,34 \pm 0,04$ & 2,82 \\
\hline 36 & 550 & 8,5 & 950 & $7,0 \pm 1,3$ & $16,0 \pm 1,7$ & $0,36 \pm 0,04$ & 3,51 \\
\hline $\mathrm{J} 7$ & 450 & 10,5 & 1.170 & $19,0 \pm 3,0$ & $14,0 \pm 1,5$ & $0,48 \pm 0,05$ & 7,02 \\
\hline 18 & 350 & 8,5 & 950 & - & - & + & - \\
\hline J9 & 500 & 11,9 & 1.200 & $40,0 \pm 5,4$ & $18,0 \pm 2,0$ & $0,81 \pm 0,08$ & 13,80 \\
\hline 310 & 500 & 10,9 & 1.190 & - & - & - & - \\
\hline J11 & 650 & 12,4 & 1.250 & $65,7 \pm 10,4$ & $13,0 \pm 1,4$ & * & 21,13 \\
\hline $\mathrm{J} 12$ & 700 & 12,7 & 1.270 & $215,0 \pm 34,4$ & $47,0 \pm 5,2$ & * & 69,53 \\
\hline $\mathrm{J} 13$ & 760 & 13,1 & 1.310 & $663,0 \pm 92,8$ & $118,0 \pm 13$ & - & 212,17 \\
\hline J13B & 900 & 12,4 & 1.240 & $167,0 \pm 26,7$ & $45,2 \pm 5,0$ & * & 54,74 \\
\hline$J 14$ & 850 & 13,1 & 1.340 & $773,0 \pm 108$ & $125,0 \pm 13,8$ & $1,4 \pm 0,15$ & 237,48 \\
\hline J15 & 1.000 & 13,6 & 1.370 & $422,0 \pm 59,0$ & $68,0 \pm 7,5$ & $*$ & 134,40 \\
\hline 117 & 750 & 11,5 & 1.200 & - & - & - & - \\
\hline $\mathrm{J} 20 \mathrm{~B}$ & 550 & 10,7 & 1.190 & - & - & - & _- \\
\hline
\end{tabular}

*Abaixo do limiar de sensibilidade $\mathrm{U}_{13 \mathrm{al}}^{238}=0,73 \mathrm{cal} \cdot \mathrm{ano}^{-1} \cdot \mathrm{g}^{-1}=0,73 \cdot 4,18 \cdot 10^{7} \mathrm{erg} \cdot \mathrm{ano}^{-1} \cdot \mathrm{g}^{-1}$ $\operatorname{Th}^{232}=0,20 \mathrm{cal} \cdot \mathrm{ano}^{-1} \cdot \mathrm{g}^{-1}=0,20 \cdot 4,18 \cdot 10^{7} \mathrm{erg} \cdot \mathrm{ano}^{-1} \cdot \mathrm{g}^{-1}$ $\mathrm{K}_{\text {nat }}=2,7 \cdot 10^{-5} \mathrm{cal} \cdot \mathrm{ano}^{-1} \cdot \mathrm{g}^{-1}=2,7 \cdot 10^{-5} \cdot 4,18 \cdot 10^{7} \mathrm{erg} \cdot$ ano $^{-1} \cdot \mathrm{g}^{-1}$

${ }^{* *}$ A dose de radiação natural, expressa $\mathrm{em}$ erg $\cdot$ ano $^{-1}$, é relativa a uma amostra de $10 \mathrm{mg}$ 
Tabela IV - Medidas de termoluminescência natural e avaliação de dose para as amostras do perfil 2, dama 2, levando-se em consideração as famílias radioativas do $\mathrm{U}^{238}, \mathrm{Th}^{232}$ e $\mathrm{K}^{40}$. Temperatura do pico de $T L$ natural $=280 \pm 2{ }^{\circ} \mathrm{C}$

\begin{tabular}{|c|c|c|c|c|c|c|c|}
\hline Amostras & $\begin{array}{c}\text { Atividade } \\
\text { (SPP3) } \\
\text { in loco }\end{array}$ & $\begin{array}{c}\text { Texmolumir } \\
\text { H (cm) }\end{array}$ & $\begin{array}{l}\text { scência natural } \\
\text { Área }\left(\mathrm{mm}^{2}\right)\end{array}$ & $\begin{array}{l}\text { Urânio } \\
\text { (ppm) }\end{array}$ & $\begin{array}{l}\text { Tório } \\
\text { (jpm) }\end{array}$ & $\begin{array}{c}\text { Potássto } \\
\text { (\%) }\end{array}$ & $\begin{array}{l}\text { Dose de radiaģão } \\
\text { natural " (ergłano) }\end{array}$ \\
\hline J1A & 300 & 6,3 & 630 & $1,2 \pm 0,2$ & $4,0 \pm 0,5$ & $0,33 \pm 0,03$ & 0,73 \\
\hline $\mathrm{J} 2 \mathrm{~A}$ & 400 & 8,0 & 720 & $4,0 \pm 0,7$ & $6,0 \pm 0,7$ & $0,32 \pm 0,03$ & 1,77 \\
\hline $\mathbf{J} 3 \mathbf{A}$ & 450 & 10,0 & 800 & $2,9 \pm 0,6$ & $18,0 \pm 2,0$ & $0,29 \pm 0,03$ & 2,42 \\
\hline J4A & 500 & 12,0 & 1.000 & $6,0 \pm 1,1$ & $11,0 \pm 1,2$ & $0,62 \pm 0,06$ & 2,82 \\
\hline $\mathrm{J} 5 \mathrm{~A}$ & 600 & 12,6 & 1.090 & $5,3 \pm 1,0$ & $15,0 \pm 1,7$ & $0,47 \pm 0,05$ & 2,92 \\
\hline J6A & 650 & 14,0 & 1.111 & $9,8 \pm 1,7$ & $16,8 \pm 1,8$ & $0,27 \pm 0,03$ & 4,43 \\
\hline J7A & 550 & 13,6 & 1.100 & $10,4 \pm 1,7$ & $17,1 \pm 1,9$ & $0,20 \pm 0,02$ & 4,63 \\
\hline $38 \mathrm{~A}$ & 560 & 15,0 & 1.150 & $13,0 \pm 2,1$ & $18,0 \pm 2,0$ & $0,32 \pm 0,03$ & 5,51 \\
\hline $99 \mathrm{~A}$ & 750 & 18,4 & 1.220 & $38,0 \pm 6,1$ & $13,0 \pm 1,4$ & * & 12,68 \\
\hline $\mathrm{J} 10 \mathrm{~A}$ & 1.100 & 20,5 & 2.030 & $136,0 \pm 22$ & $34,0 \pm 3,7$ & - & 44,34 \\
\hline J11A & 800 & 19,0 & 1.800 & - & - & - & - \\
\hline $312 \mathrm{~A}$ & 800 & 19,3 & 1.810 & - & - & - & - \\
\hline $\mathrm{J13A}$ & 800 & 19,3 & 1.800 & - & - & - & - \\
\hline J14A & 850 & 19,6 & 1.880 & - & - & - & - \\
\hline J15A & 900 & 20,1 & 2.000 & - & - & - & - \\
\hline
\end{tabular}

* Abaixo do limiar de sensibilidade

$$
\begin{aligned}
& \begin{aligned}
\mathrm{U}_{n a t}^{238} & =0,73 \mathrm{cal} \cdot \mathrm{ano}^{-1} \cdot \mathrm{g}^{-1}=0,73 \cdot 4,18 \cdot 10^{7} \mathrm{erg} \cdot \mathrm{ano}^{-1} \cdot \mathrm{g}^{-1} \\
\mathrm{Th}^{232} & =0,20 \mathrm{cal} \cdot \mathrm{ano}^{-1} \cdot \mathrm{g}^{-1}=0,20 \cdot 4,18 \cdot 10^{7} \mathrm{erg} \cdot \mathrm{ano}^{-1} \cdot \mathrm{g}^{-1}
\end{aligned} \\
& \mathrm{~K}_{\text {nat }}=2 ; 7, \cdot 10^{-5} \mathrm{cal} \cdot \mathrm{ano}^{-1} \cdot \mathrm{g}^{-1}=2 ; 7 \cdot 10^{-5} \cdot 4,18 \cdot 10^{7} \mathrm{erg} \cdot \mathrm{ano}^{-1} \cdot \mathrm{g}^{-1}
\end{aligned}
$$

Estudando as correlações existentes entre a $T L$ natural e as variações nas concentrações de urânio (ppm) em função da distância ao nível mineralizado, cluas conclusões podem ser tiradas. A $T L$ natural vérsus a distância ao nível mineralizado, parece uma função quase que linear, enquanto que as concentrações de urânio decrescem muito rapidamente. Pode-se observar ainda que a diminuição da $T L$ em relação às concentrações de urânio é bem mais lenta, mostrando a não-existência de uma correlação direta (Fig. 5).

Tais comportamentos anômalos podem ser melhor entendidos levando-se em conta os seguintes aspectos. Para um meio não-homogêneo, como o do caso em questão, o urânio desempenha um papel importante na irradiação interna das amostras; parte desta é originada pelo emissores gama localizados fora da mesma. Nessas condições, a maior parte da amostra é irradiada pelos emissores de curto alcance, assim como pela radiação gama externa, que é emitida por uma fonte bastante intensa. Para pontos afastados do nível mineralizado, observa-se um decréscimo. da $T L$, correspondente a várias energias emitidas pelos descendentes do urânio. Por outro lado, a correlação entre a $T L$ natural e a atividade gama total parece aceitável para contagens gama inferiores a $600 \mathrm{~Hz}$, mostrando, no entanto, uma tendência à saturação para valores superiores a esse. Isso explica satisfatoriamente o decréscimo da intensidade da $T L$ em função da distância ao filão mineralizado.

TERMOLUMINESCENCIA NATURAL EM FUNÇÃO DA INTENSIDADE DE DOSE ABSORVIDA Com base nas determinações dos principais elementos radioativos (urânio, tório e potássio), por espectrometria gama em laboratório, calculou-se a intensidade de dose absorvida, em erg ano $^{-1}$, correspondente a $10 \mathrm{mg}$ de amostra. Os efeitos devido à radiação cósmica não foram levados em conta, uma vez que a rocha em 


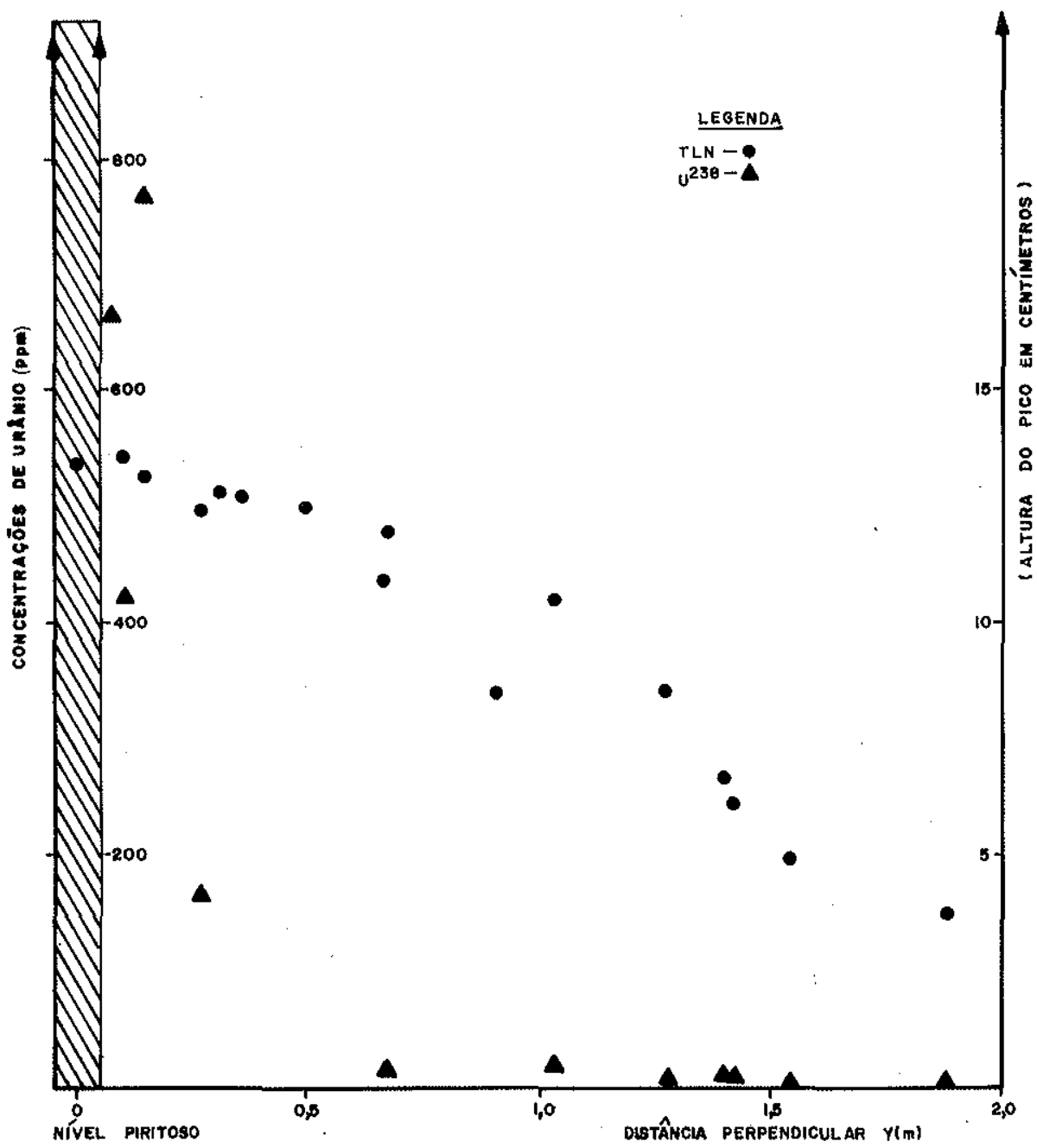

Figura 5 - Variações da termoluminescência natural e das concentrações de urânio (ppm) em função da distância ao nível piritoso

estudo continha mais de $1 \mathrm{ppm}$ de urânio e tório. Da mesma forma, os efeitos produzidos pela radiação gama externa à mesma não foram consideradas, pois, esta só interfere de modo apreciável até $0,50 \mathrm{~m}$ do filão mineralizado (Fig. 5). $O$ estudo da $T L$ natural em função da intensidade de dose absorvida mostrou um crescimento quase que linear da $T L$ para pequenas doses e, acima de certo valor, uma região de saturação (Fig. 6).

Sabe-se que, quando uma substância exibe o fenômeno da $T L$, restrições específicas à temperatura são mantidas durante a excitação dos elétrons. No caso em questão, considerando-se os aspectos geológicos que determinaram a formação dos quartzitos, bem como a deposição da camada mineralizada, certamente tais fenômenos influenciaram a acumulação da TL. Também o gradiente de calor provocado quando da deposição da camada mineralizada drenou a $T L$ preexistente, supondomse, entretanto, que a radiação natural preencheu posteriormente os defeitos já existentes e criou outros adicionais. 




Figura 6 - Termoluminescência natural em função da intensidade de dose de radiaçãa absorvida, para iguais pesos de amostras $(10 \mathrm{mg})$

ESTIMATIVA E INTERPRETAÇÃO DO TEMPO DE AGUMULAÇÃO DA TERMOLUMINESGENCIA NATURĀL NO QUARTZITO O fenômeno da TL é uma função que depende da acumúlação da energia nos cristais, e do tempo. Existem casos em que o tempo de acumulação da $T L$ a que foram submetidas as amostras não representa idade geológica das mesmas, a exemplo do que acontece com rochas metamórficas (Johńnson, 1963). Para o caso em questão, o tempo que se pode tentar estimar refere-se a estágios subsaturados da $T L$ à temperatura constante, ou pelo menos consideravelmente inferior à do pico de $T L$ natural detetado, $280 \pm 2{ }^{\circ} \mathrm{C}$.

A curva de intensidade da $T L$ vérsus dose absorvida foi utilizada na tentativa de datar o evento geológico que deu origem à acumulação da $T L$ no quartzito, utilizando-se apenas sua região linear. Por outro lado, sendo os quartzitos pouco fraturados e não alterados, pode-şe considerá-los como um sistema fechado conveniente para datação. Tal consideração permite que se admita que o urânio se encontre possivelmente em equilíbrio radioativo com seus descendentes, pois não houve migrações de rádio ou radônio em porcentagens apreciáveis (Aitken, 1968). Dessa forma, tomando-se a região linear da Fig. 6, pode-se definir que a $T L$ natural é função da intensidade de dose absorvida $(I)$, relativa a uma amostra de $10 \mathrm{mg}$ e expressa $\mathrm{em} \mathrm{erg} \cdot \mathrm{s}^{-1}$, do rendimento de acumulação da $T L$ $(R)$, e do tempo. Quanto à $T L$ artificial, depende da dosé equivalente, além do rendimento de acumulação, tal que:

$$
t=\frac{D(\text { dose artificial })}{I \text { (natural) }} \text { (anos). }
$$

As Tabs. V e VI mostram os resultados obtidos a partir dessa expressão, nas quais se observa que as amostras exibem idades diferentes, mas de um modo geral, da mesma ordem de grandeza. 
Tabela V - Avaliações da intensidade de doses absorvidas natural e artificial - perfil 1, dama 1 , considerando-se as famílias radioativas do $\mathrm{U}^{238}, \mathrm{Th}^{232}$ e $\mathrm{K}^{40}$. Temperatura do pico de $T L$ natural $=280 \pm 2{ }^{\circ} \mathrm{C}$.

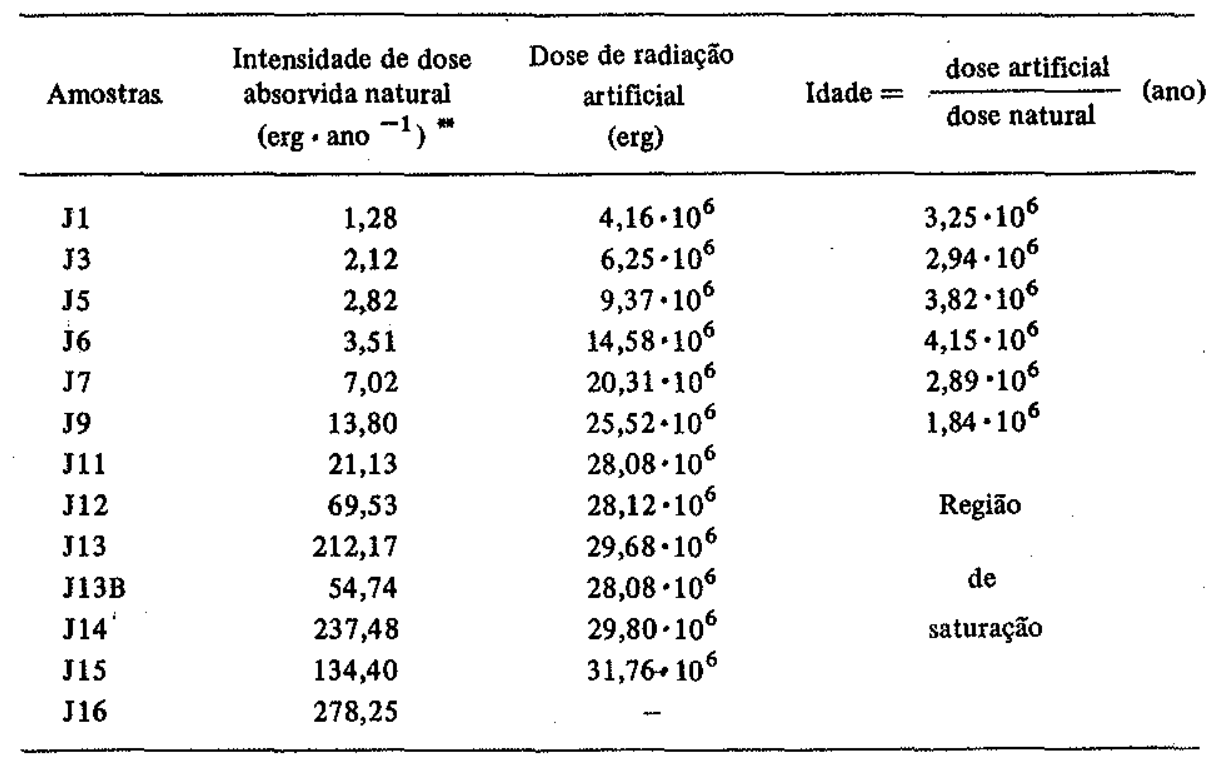

$\mathrm{U}_{\mathrm{nal}}^{238}=0,73 \mathrm{cal} \cdot \mathrm{ano}^{-1} \cdot \mathrm{g}^{-1}=0,73 \cdot 4,18 \cdot 10^{7} \mathrm{erg} \cdot \mathrm{ano}^{-1} \cdot \mathrm{g}^{-1}$

$\mathrm{Th}^{2} \mathrm{H}^{2}=0,20 \mathrm{cal} \cdot \mathrm{ano}^{-1} \cdot \mathrm{g}^{-1}=0,20 \cdot 4,18 \cdot 10^{7} \mathrm{erg} \cdot \mathrm{ano}^{-1} \cdot \mathrm{g}^{-1}$

$\mathbf{K}_{\text {rat }}=2,7 \cdot 10^{-5} \mathrm{cal} \cdot \mathrm{ano}^{-1} \cdot \mathrm{g}^{-1}=2,7 \cdot 4,18 \cdot 10^{2} \mathrm{erg} \cdot$ ano $^{-1} \cdot \mathrm{g}^{-1}$ de $10 \mathrm{mg}$

**A intensidade de dose absorvida natural, expressa $\mathrm{em}$ erg $\cdot$ ano ${ }^{-1}$, é relativa a uma amostra

CONCLUSŌES A interpretação dos perfis através da $\%$ natural mostrou um decréscimo quase que linear em função da distância ao nível mineralizado, embora para pontos próximos a este, onde a concentração dos elementos radioativos revelou-se bastante elevada, a $T L$ exibida pelo quartzito apresentou o fenômeno de saturação. Semelhantemente, observou-se que a atividade gama total medida no interior da mina decresceu em relação aos pontos afastados do nível piritoso e, além disso, uma correlação positiva entre a $T L$ natural e os teores de urânio foi verificada. Trabalhos efetuados por White (1957), em Canavieiras, mostraram existir uma correlação direta entre minerais radioativos e ouro, enquanto que aqui foi possível mostrar que pontos dos perfis de baixa concentração exibem resposta termoluminescente também de baixa intensidade. Para que uma determinada substância apresente o fenômeno da $T L$, torna-se necessário que restrições específicas à temperatura tenham sido mantidas durante a excitação dos elétrons. É possível que diferentes pontos da rocha ora estudada tenham sido submetidos a diferentes aquecimentos, de modo tal que o maior dos valores calculados representa uma idade mínima que pode caracterizar um evento geológico relativamente recente. Fenômeno idêntico pode também ocorrer quando se procura estabelecer idades de rochas através do método K-Ar. Nessas condições, o valor de $12,1 \pm 0,3$ m.a. será aqui utilizado como indicativo de uma idade mínima que poderá ser interpretada de duas maneiras:

1) representar um evento geológico de idade miocênica;

2) representar uma idade mínima sem qualquer conotação com a história térmica da rocha. 
Através dos dados da geologia regional e de outros dados geocronológicos, não temos conhecimento de eventos ocorridos no Mioceno, de modo que teremos de admitir a idade de $12,1 \pm 0,3$ m.a. como resultante da liberação quase total dos elétrons armadilhados e, provavelmente, ligada a um intemperismo acentuado.

Tabela VI - Avaliações da intensidade de doses absorvidas natural e artificial perfil 2, dama. 2, considerando-se as famílias radioativas do $\mathrm{U}^{238}, \mathrm{Th}^{232} \mathrm{e} \mathrm{K}^{40}$. Temperatura do pico de $T L$ natural $=280 \pm 2{ }^{\circ} \mathrm{C}$

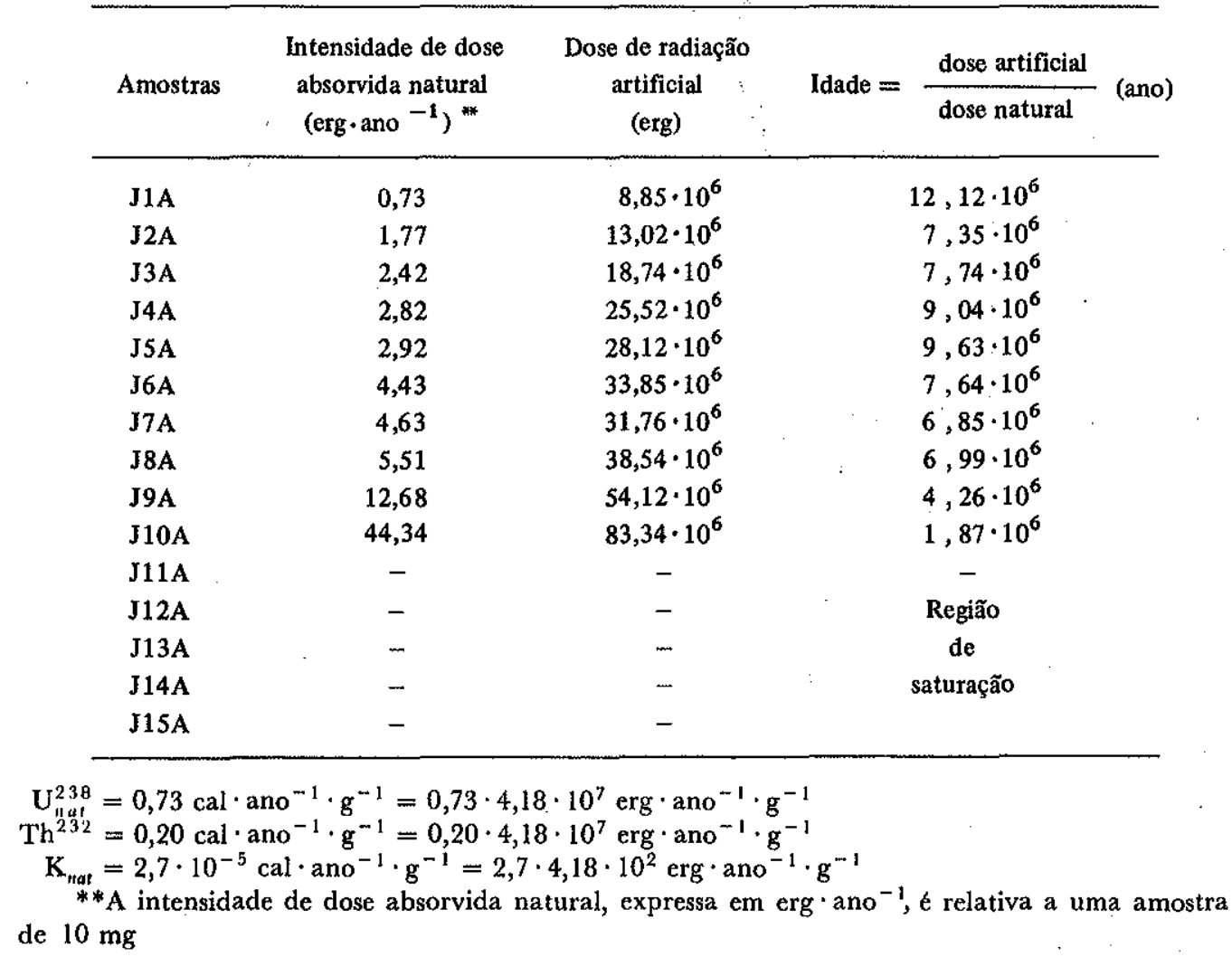

\section{BIBLIOGRAFIA}

AITKEN, M. J. - 1968 - Thermoluminescent dating in Archeology. Thermoluminescence of Geological Materials Academic Press, London and New York, pp. 369-377

BATEMAN, J. D. - 1958 - Uranium bearing auriferous reefs at Jacobina Brazil. Econ. Geol. 53: $417-425$

GULLITY, B. D. - 1967 - Elements of X-ray difraction. Addison Wesley Publishing Company Inc., London

FERREIRA, J. - 1973 - Estudo da termoluminescência do Quartzito em uma zona mineralizada em ouro e uranio (Canavieiras, Jacobina, Bahia). de Mestrado, Universidade Federal da Bahia (inédito) 
JOHNSON, N. M. - 1963 - Thermoluminescence in contact metamorphosed limestone, J. Geol. 71: $596-616$

NORDEMANN, D. - 1966 - Emissions gamma de quelques méteorites et roches terrestres. Evaluation de la radioactivité du sol lunaire. Thèse, Paris (inédito)

WHITE, M. G. - 1957 - Uranium in the Serra Jacobina, State of Bahia, Brazil, United Internat. Conf. Peaceful. Usis of Atomic Energy Geneva, 1955. Proc. 6, 140 pp.

WHITE, M. G. - 1964 - Urânio em Morro do Vento, Serra de Jacobina, Bahia. Geological Survey Bulletin, 185-A 